\title{
The Effect of Taping on Foot Structure, Functional Foot Stability and Running Gait Patterns of the Foot
}

\author{
Malia Ho Tsai Djun and John Cher Chay Tan \\ Department of Physical Education and Sports Science, Nanyang Technological University, Singapore 637616, Singapore
}

\begin{abstract}
Running related foot injuries are associated with excessive foot movements due to malaligned foot structure and poor functional foot stability. Clinicians tape the foot to alleviate pain and prevent further injuries, whilst allowing the athlete to continue training. However, the effect of taping is not conclusive. The purpose of this study is to investigate if taping effectively improves foot structure, functional foot stability and reduces excessive foot movements during running. Twenty-two subjects had their foot structure identified as: flat foot stable, flat foot unstable and normal arched unstable according to the FPI (foot posture index) and the Modified Romberg's Test with the BESS (balance error scoring system) criteria. The subjects ran on an instrumented treadmill barefooted with their feet taped and untaped. Running kinetic and kinematic data were collected and analysed using a paired t-test and $3 \times 2$ ANOVA. Taping improved foot structure but not functional foot stability. During running, taping significantly reduced rearfoot eversion. Taping increased the loading rate in the flat foot and normal arched unstable groups but reduced the loading rate for the flat foot stable group. Implication on the appropriate use of foot taping was discussed.
\end{abstract}

Key words: Running related injuries, flat feet, functional foot stability, three dimensional motion analysis, foot taping, instrumented treadmill

\section{Introduction}

Foot injuries are usually attributed to malaligned foot structure, such as flat feet [1] and/or poor functional foot stability [2].

\subsection{Foot Structure and Injuries}

The normal arched foot is considered to be structurally aligned. The flat foot is defined as a foot that is in an over-pronated position when weight bearing and is considered to be structurally malaligned compared to the normal arched foot [3]. The flat foot is thought to exhibit a more pronated gait, characterized by excessive foot eversion, flattening of the medial longitudinal arch and limited first toe dorsiflexion. These deviated foot movements have been thought to be the pre-cursor to foot injuries. Some individuals with flat foot structures have thus been discouraged from sports as they have been

Corresponding author: Malia Ho Tsai Djun, M.S., research field: biomechanics of the lower limb. E-mail: maliahotd@gmail.com. thought to be at a higher risk of foot injuries.

\subsection{Functional Foot Stability and Injuries}

Functional foot stability is defined in this study as "the ability of the foot to continually adjust its position to maintain the body in an upright, balanced position". This is based on the work of Freeman, Dean and Hanham [4] who stated that individuals with functionally unstable feet would present with balance deficiencies when standing on one leg. Feet with good functional stability would have good position sense and good muscular control [5]. During locomotion, an individual with good functional foot stability would be able to continually adjust the position of his foot relative to the ground to reduce excessive movements. These excessive movements may be pre-cursors to foot injuries [6]. Individuals with functional foot instability do not have this protective ability and could also be more prone to foot injuries. 


\subsection{Effects of Taping}

In treating foot injuries, clinicians seek to improve foot structure and functional foot stability. Low-Dye taping of the foot is one of the modalities used routinely to improve foot structure and improve foot stability in order to prevent further injury, without too much restriction in activity [7]. However, the effect of taping the foot is unclear. Herradine, Herrington and Wright [8] found that taping improved foot structure significantly by reducing the standing calcaneal eversion from $6^{\circ}$ to $4^{\circ}$. Cornwall, McPoil and Fair [9] found that taping increased arch height by $2 \mathrm{~mm}$. However, this effect was lost after $3.2 \mathrm{~km}$ of treadmill running. Luque-Suarez, Gijon-Nogueron, Baron-Lopez, Labajos-Manzanares, Hush and Hancock [10] reported that taping did not improve foot structure.

It is believed that taping provides a traction tension on the skin which stimulates the sensory receptors and aids position awareness [11]. The ability to continually adjust the foot position to maintain balance depends largely on the ability of the foot to sense and improve its position. Better position sense would be associated with better functional foot stability. Robbins, Waked and Rappel [12] made their subjects stand on blocks with different inclinations and measured their position sense before and after taping. They concluded that the taped feet were able to estimate the inclination better than when they were untaped. However, other studies [13, 14] did not support the effect of taping improving functional foot stability as it was reported that taping did not improve balance ability $[13,14]$ and muscular activation in the foot and ankle during one legged stance tests [14].

The effect of taping in reducing excessive movements during gait is also inconsistent. Stoffel, Nicholls, Winata, Dempsey, Boyle and Llyod [15] found that range of motion of the rearfoot was effectively reduced by taping during running. Vicenzino, McPoil and Buckland [16] also supported this when they observed that taping increased the plantar pressure on the lateral side of the midfoot during jogging, inferring that there was less rearfoot eversion. O'Sullivan, Kennedy, O'Neill and $\mathrm{Ni}$ Mhainin [17] reported that the main effect of low-Dye taping was to reduce rearfoot eversion movements during walking but had no effect on the arch profile of the foot. However, Herradine et al. [8] found that taping had no effect on rearfoot eversion during running. Stoffel et al. [15] found that taping did not reduce the range of motion of the foot-ankle joint complex during sporting tasks. Although the efficacy of taping the foot remains questionable, taping continues to be advocated for use as a modality for injury management [7].

The reasons for the difference in results could be due to the type of activity measured (for example, running or walking), the difference in the sample population (injured foot or non injured foot, flat foot or normal arched foot) and the taping technique used (low-Dye taping or figure-of-eight taping).

Among many techniques and variations of foot taping, Carter and Chockalingam [18] compared two low-Dye and two high-Dye taping techniques and found that the ALD (augmented low-Dye) taping technique was most efficient in increasing the plantar pressure on the lateral aspect of the foot during walking. It would be of injury management interest to examine the effect of ALD taping technique on running.

\subsection{Purpose}

The purpose of this study was to investigate if ALD taping improved foot structure, functional foot stability and if it augmented foot movements and contact forces in running. The results of this study will indicate which foot pathologies will be appropriately managed with ALD taping and which group of individuals would benefit most from ALD. If the results of this study showed that ALD taping was effective, then it should be used as a protective gear for individuals who may be more prone to foot related 
running injuries such as individuals with flat feet and poor functional foot stability.

If ALD taping was not found to be effective, then the continued use of ALD taping should be discouraged as runners who tape their feet would have a false sense of security, thinking their feet were protected when they were actually not. This would potentially expose them to further foot injuries.

\section{Method}

\subsection{Subject Recruitment and Characteristics}

All procedures were approved by the IRB (Institutional Review Board) of the Nanyang Technological University (Singapore). It was ensured that all subjects did not have foot or ankle injuries in the last six months, foot or ankle disease or abnormalities that may affect gait and balance. Such conditions may hinder subjects from completing the running trials. Details of the research study were described to the subjects before they signed the consent form as required by the IRB.

Twenty-two subjects (age $=24.9 \pm 3.8$ years, body mass index $=22.7 \pm 3.0 \mathrm{~kg} \cdot \mathrm{m}^{-2}$ ) made up of 9 females and 13 males participated in this study. Each foot was taken as a functional unit yielding a total of 44 feet. The subjects were required to attend a single session where they completed two running trials with their feet taped and untaped.

\subsection{Measure of Foot Structure}

The FPI (foot posture index) has been found to be a reliable and valid tool to assess foot structure [19, 20]. It has also been shown to be able to predict dynamic plantar pressures within the three foot structures [21]. The FPI was conducted according to the Foot Posture Index User Guide and Manual [22]. The criteria assessed included: palpation of the talar head, observations of the curves above and below the lateral malleoli, the extent of inversion and eversion of the calcaneus, assessing the medial bulge of the Talo-navicular joint, the congruence of the inner longitudinal arch and the extent of adduction or abduction of the forefoot. Each criterion was scored on a Likert-type scale from -2 to +2 . The scores for all six criteria were added up to obtain a composite score of between -12 to +12 . A normal arched foot would have an FPI score of between 0 and 5 , while a flat foot would have a score of +6 to +12 . A high arched foot would have a score of -12 to -1 .

\subsection{Measure of Functional Foot Stability}

Functional foot stability is usually tested clinically with the Modified Romberg's test, based on the work of Freeman et al. [4]. In this study, the criteria set out by the BESS (Balance Error Scoring System) were used to quantify the subject's quality of balance [23]. Although the BESS tests postural balance under a variety of conditions, the firm surface, single leg component of the BESS has been found to be as effective as quantitative force plate measures and was found to be a reliable field test to measure foot and ankle stability [24].

To ascertain the functional stability of the feet in this study, the subjects were asked to stand on the test limb with their eyes closed and hands on their iliac crests for 20 seconds. The number of BEs (balance errors) were counted and recorded. The BEs were lifting hands off the hips, opening of eyes, abduction or flexion of hip more than $30^{\circ}$, lifting of forefoot or heel of the weight bearing foot, remaining out of the proper testing position for more than 5 seconds and placing the non-weight bearing foot on the ground. The higher the number of balance errors, the more functionally unstable the foot was deemed [25]. If two BEs were committed at the same time, they were only counted as one BE. A foot with less than two BEs was considered functionally stable while a foot with more than three BEs was considered functionally unstable.

\subsection{Test Reliability}

To eliminate the risk of errors due to multiple assessors, the measures of all the feet were scored by a 
single assessor $\mathrm{MH}$ (Malia Ho) with 16 years of clinical experience. As the FPI and BE were subjective tests, a study was done to ascertain the reliability of $\mathrm{MH}$ in performing the tests of measuring foot structure and functional foot stability. $\mathrm{MH}$ measured the FPI and BE of eight subjects on two separate occasions and the ICC (intra-rater correlation coefficient) for FPI was excellent (ICC $=0.92,95 \%$ $\mathrm{CI}=0.75-0.97)$. The ICC for BE was good (ICC = $0.86,95 \% \mathrm{CI}=0.64-0.96)$. This showed that $\mathrm{MH}$ was a reliable assessor. The order of foot structure and functional foot stability tests were randomised to reduce biasness.

\subsection{Subject Preparation and ALD Taping}

The subjects' feet were washed to ensure maximum tape adhesion. The same tester (MH) applied reflective markers and the tape to all the subjects' feet.

Twenty-two reflective markers were used and they were positioned on the subject's lateral fibula head, medial tibial condyle, lateral and medial malleolus, medial and lateral aspect of the calcaneus, navicular tuberosity, the styloid process of the 5th metatarsal, first and fifth MPJ (metatarsophalangel joints) and the medial aspect of the distal phalanx of the first toe.

To reduce subject bias, the expected outcome of taping the foot was not communicated to the participants. The order of the taped and untaped trials was randomised. Therefore, some subjects performed the trials with their feet untaped first and then taped, whilst some subjects performed the trials with their feet taped first and then untaped.

Rigid sports tape (Endura) of width $2.5 \mathrm{~cm}$ was used to tape the foot. A strip of tape was first wrapped round the leg slightly above the medial and lateral malleolus. Another strip of tape was then brought from the anterior of the ankle, round the medial aspect of the ankle, inferiorly round the lateral ankle, medially up the inner longitudinal arch and finally brought up to the anterior aspect of the ankle again. Three strips were used. Fig. 1 shows the taping process with only two strips placed. Finally, tape was placed round the ankle to secure the ends of the strips.

The subject was then asked to stand up to ensure that the tape was relatively comfortable before the trial was conducted.

\subsection{Data Collection}

Running gait data were recorded with six optical Hawk cameras (Motion Analysis Corporation, Santa Rosa, CA, USA) placed strategically around a Gaitway Kistler Instrumented Treadmill (Kistler Corporation, USA). This set up allowed the capture of kinematic and kinetic data from multiple consecutive steps.

The subject stood on the treadmill within the capture volume and a static trial was captured. The position data of the markers were digitised using the Cortex software (Motion Analysis Corporation, Santa Rosa, CA, USA).

The treadmill was then started and the speed was increased until the subjects started running. This was characterised by a presence of a flight phase where both feet were not in contact with the treadmill belt. As running speed may affect gait parameters, the speed was kept within a narrow range of 1.4-2.2 $\mathrm{ms}^{-1}$ and all the subjects ran with a heel strike running gait, characterised by the heel striking the ground first. There was a warm-up run of 6 minutes before collection of the kinematic and kinetic data collection

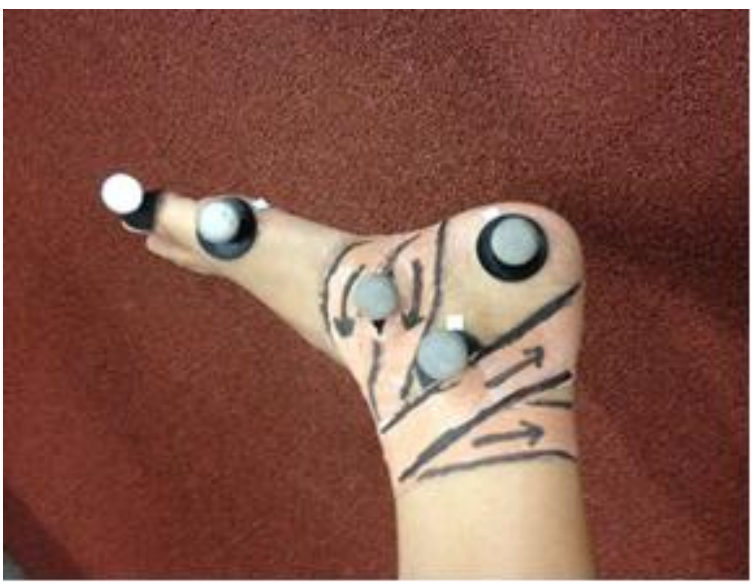

Fig. 1 ALD taping technique used in this study. 
commenced. Running data were collected for 10 seconds.

\subsection{Data Processing}

Five consecutive steps were selected for analysis. Each kinematic trial data collected from the Cortex Software was visually checked and gaps in the trials were filled with a cubic spline. The data were then filtered using a Butterworth low pass, 4th order, zero phase filter at $10 \mathrm{~Hz}$ as recommended by Kirtley [26].

This data was then exported in C3D format and joint movements were measured using the Visual 3D software (C-motion Inc, Germantown, MD, USA). The four rigid segments consisting of the leg, rearfoot, midfoot and first toe were modelled as simple geometric solids with uniform densities as seen in Fig. 2.

\subsection{Dependant Variables}

Pronatory movements are characterised by observing the movement of the rearfoot eversion, arch flattening and first toe dorsiflexion [27]. The total excursion of the rearfoot eversion was measured as the movement of the rearfoot with reference to the leg in the coronal plane. The amount of arch flattening is measured by the total excursion of midfoot dorsiflexion with reference to the rearfoot in the sagittal plane and total excursion of the first toedorsiflexion were measured as the movement between the first toe with reference to the midfoot segment in the sagittal plane.

Kinetic variables collected by the Gaitway instrumented treadmill include maximum vertical ground reaction force (vGRFmax) and loading rate. vGRFmax is the maximum ground reaction force pushing against the foot during running. Loading rate is defined as the rate of change of vertical ground reaction force over time during heel strike. These variables were selected as they have been thought to be relevant components contributing to running related injuries $[28,29]$. The kinetic variables were normalised to the subject's body weight. The mean values for the five selected steps were time-normalised to the weight bearing phase of gait.

\subsection{Statistical Analysis}

To analyze the effect of taping on foot structure, functional foot stability and running gait, a paired t-test was first used to compare this variables with the foot taped and untaped.

The 44 feet were divided into three groups: FFS (flat foot stable), NUS (normal arched unstable) and FFUS (flat foot unstable). There were 13 feet in the FFS group, 15 feet in the NUS group and 16 feet in the FFUS group. The data were analysed comparing the sample population with their feet taped and untaped. The FFS group would elucidate the effect of taping on individuals with flat feet only, the NUS group would elucidate the effect of taping on individuals with functional foot instability only, and finally, the FFUS group would elucidate the effect of taping on individuals who had flat feet and functional foot instability.

A $3 \times 2$ mixed ANOVA was used to study the differences between groups (FFS, NUS and FFUS) and between conditions (taped and untaped).

\section{Results}

\subsection{Pairwise Comparison}

The effect of taping on the foot structure (FPI scores)

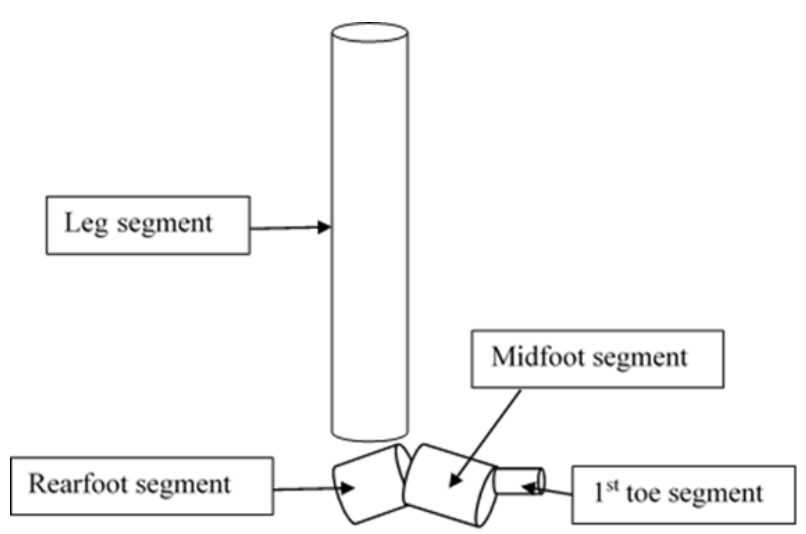

Fig. 2 Model of right foot and leg created in V3D software. 
and functional foot stability (BE scores) of the 44 feet are shown in Table 1.

The results showed that taping improved foot structure significantly. Taping had no statistically significant effect on BE scores.

The effect of taping on running gait parametersis shown in Table 2

The results in Table 2 show that taping reduced rearfoot eversion significantly during running but had no significant effect on the other variables.

\subsection{Group Comparison}

The results were also analysed by groups using a three-by-two mixed ANOVA. Figs. 3 and 4 show the effect of taping on foot structure and functional foot stability between the three groups. The solid line represents the untaped condition while the dashed line represents the taped condition. If the two lines are lying one on top of the other, it shows that taping has no effect on that particular dependant variable.

From Fig. 3, the reduction in FPI was significant across all three groups $(\mathrm{F}(1,42)=28.739, P=0.000)$. There was no significant interaction between the tape conditions and groups $(\mathrm{F}(2,41)=1.977, P=0.151)$. The result of a series of paired t-tests indicated that taping significantly reduced the FPI of the FFUS and FFS groups only $(P<0.05)$.

From Fig. 4, it was noted that the difference in error score was not significant across all three groups $(\mathrm{F}(1$, $42)=0.516, P=0.476)$. There was also no significant interaction between tape conditions and groups $(\mathrm{F}(2$, 41) $=1.149, P=0.327)$. However, paired t-tests between taped and untaped conditions showed that the BE score was significantly higher in the FFS group $(P$ $<0.05)$.

Figs. 5-9 illustrated the effect of taping on the running gait variables.

Fig. 5 showed that the reduction in rearfoot eversion was significant. $(\mathrm{F}(1,42)=5.901, P=0.020)$. There was no significant interation effect between the taping conditions and groups $(\mathrm{F}(2,41)=1.634, P=$ 0.208). However, paired t-tests revealed that rearfoot eversion was significantly reduced in the FFS group only $(P<0.05)$.

There was no significant difference in movement of the midfoot dorsiflexion due to taping amongst the three groups $(\mathrm{F}(1,42)=2.646, P=0.111)$. There was no interaction effect between taping conditions and groups $(\mathrm{F}(2,41)=1.398, P=0.259)$. Paired t-tests also did not show a difference due to taping within groups.

There was also no significant difference in 1 st toe dorsiflexion due to taping amongst the three groups $(\mathrm{F}(1,42)=0.1 .755, P=0.193)$. There was no significant interaction effect between taping conditions and groups $(\mathrm{F}(2,41)=0.272, P=0.763)$. Paired t-tests also showed no difference within groups.

Table 1 Pairwise comparison of foot structure and functional foot stability between untaped and taped conditions.

\begin{tabular}{llll}
\hline Variable & Untaped & Taped & Mean difference \\
\hline FPI (SD) & $5.8(3.0)$ & $4.7(3.1)$ & $-1.1 * * *$ \\
BE (SD) & $3.1(1.9)$ & $3.3(1.9)$ & 0.2 \\
\hline
\end{tabular}

$* * * P<0.00$

Table 2 Pairwise comparison of running gait variables between untaped and taped conditions.

\begin{tabular}{llll}
\hline Variable & Untaped & Tape & Mean difference \\
\hline Rearfoot eversion/degrees (SD) & 7.8 & 7.1 & $0.7^{*}$ \\
Midfoot dorsiflex-ion/degrees & $(1.9)$ & $(2.1)$ & 0.6 \\
(SD) & 8.7 & 9.3 & $(2.9)$ \\
1st toe dorsiflex-ion/degrees & $(2.7)$ & 8.4 & -1.0 \\
(SD) & 9.4 & $(5.4)$ & 0.0 \\
vGRFmax/per body weight (SD) & $(5.3)$ & 2.0 & $(0.2)$ \\
& $(0.2)$ & 20.7 & 0.4 \\
\hline
\end{tabular}

$* P<0.05$. 


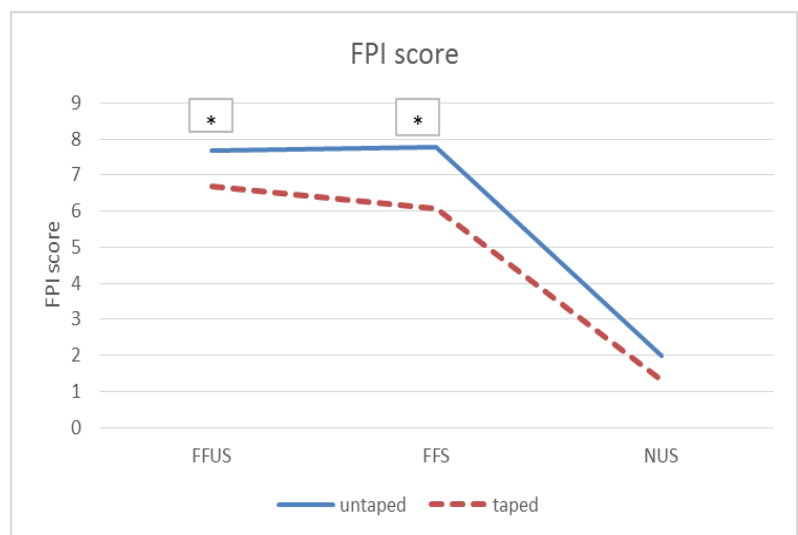

Fig. 3 FPI between taped and untaped conditions $* P<$ 0.05 .

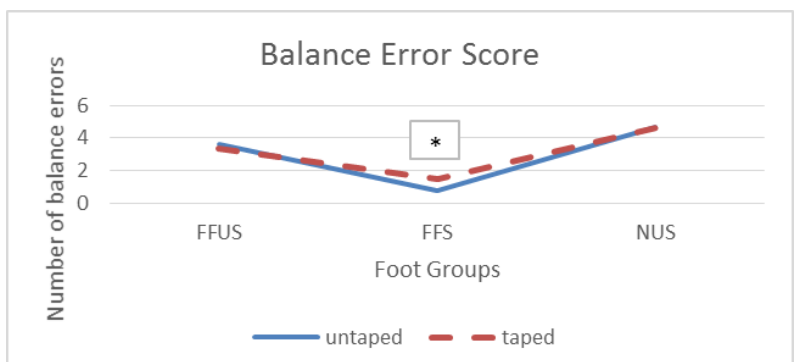

Fig. 4 BE Score between taped and untaped conditions $* P$ $<0.05$.

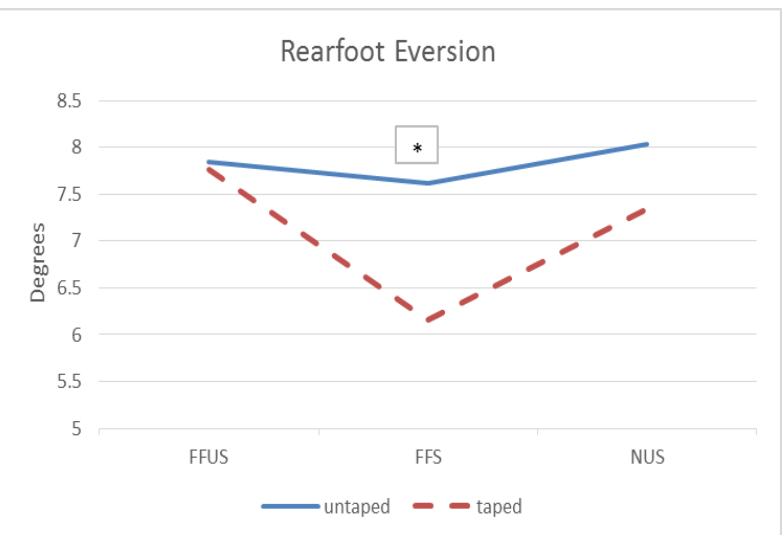

Fig. 5 Rearfoot eversion between taped and untaped conditions $* \boldsymbol{P}<0.05$.

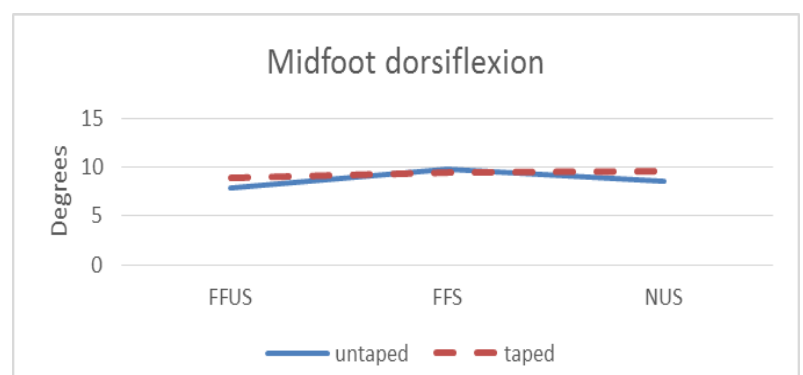

Fig. 6 Midfoot dorsiflexion between taped and untaped conditions.

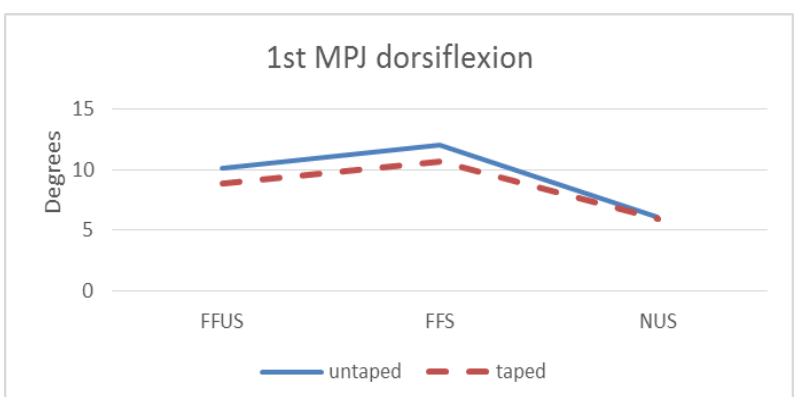

Fig. 7 First toe dorsiflexion between taped and untaped conditions.

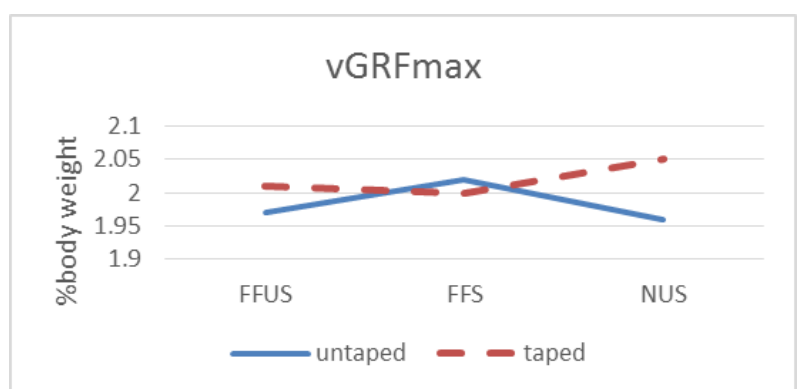

Fig. 8 Maximum vertical ground reaction force (vGRFmax) between taped and untaped conditions.

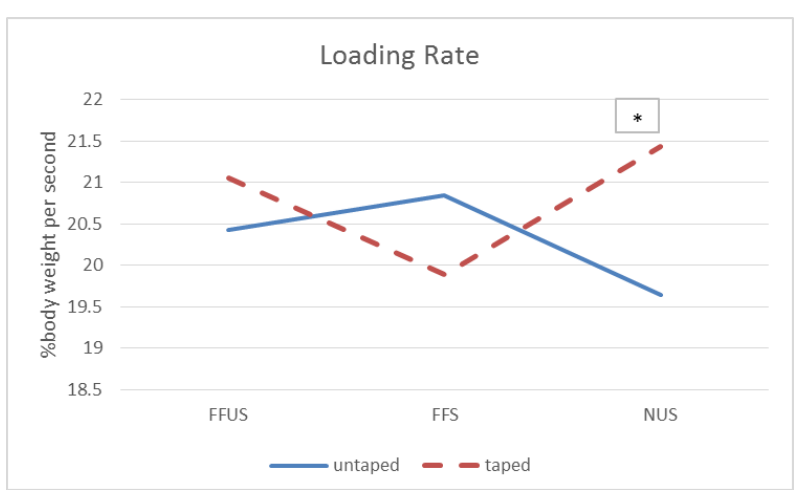

Fig. 9 Loading rate between taped and untaped conditions $* P<0.05$.

Fig. 8 showed that taping did not significantly alter the vGRFmax amongst the three groups $(\mathrm{F}(1,42)=$ 2.226, $P=0.143)$. There was no interaction effect between taping conditions and groups $(\mathrm{F}(2,41)=$ 1.170, $P=0.320)$. Paired t-tests indicated no difference due to taping within groups.

There was no significant difference in the loading rate due to taping amongst the three groups $(\mathrm{F}(1,42)=$ $1.441, P=0.237)$. However, there was a significant interaction effect of taping for loading rate $(\mathrm{F}(2,42)=$ 5.160, $P=0.01)$. Paired t-tests showed that the NUS group had higher loading rate after the foot was taped 
$(P<0.05)$ and the t-test for the FFS group approached significance $(P=0.06)$.

\section{Discussion}

\subsection{The Effect of Low-Dye Taping on Foot Structure}

The results of this study showed that low-Dye taping improved foot structure. This was supported by the significantly lower FPI scores after taping the feet. Table 1 showed that the mean FPI scores lowered from 5.8 to 4.7 changing the foot classification from a flat foot to a normal arched foot. Analysing the data by groups, taping was found to improve foot structure significantly in the flat foot subjects only (Fig. 3). This agrees with the findings of previous studies $[8,9]$ reporting a significant increase in arch height immediately after low-Dye taping of the foot. The way the tape was placed in this study was to provide external rigid support to invert the heel and hold up the arch. The rigid tape used does not stretch or lengthen, thus effectively limiting the heel eversion and arch collapse when the subject was standing. Luque-Suarez et al. [10], however, found no improvement in standing foot posture. This could be because they used elastic tape instead of rigid tape. Further studies are recommended to explore the effect on the foot structure when tapes of different materials are used.

\subsection{The Effect of Low-Dye Taping on Functional Foot Stability}

The results of our study showed that low-Dye taping did not improve functional foot stability. The results of this study concurred with the findings of Delahunt, McGrath, Doran and Coughlan [12] who found no actual increase in functional foot stability in their subjects with taping the foot with rigid tape. However, when the data were analysed by groups (Fig. 4), taping was shown to cause the FFS group to become less stable. This was an interesting finding as taping of the foot was not expected to reduce functional foot stability.
One possible explanation may be that an individual may have adapted his neuromuscular control to his foot structure. In this study, taping was found to improve the foot structure of those with flat feet but not those with normal arched feet. When the foot structure of the FFUS and FFS groups were changed by taping, the individual would have to re-adapt his sensory and motor function to the new position of the foot. This sudden need for adaptation may have produced a greater demand for muscular control [30] and may produce a transient lack of neuromuscular control to maintain stability. FFUS group may not possess this neuromuscular ability in the first place and this could be the reason why only the FFS group exhibited more balance errors when the foot was taped.

The results of this study indicated that the main function of taping was to restrict excessive movement of the foot. Studies [30, 31] reported that subjects perceived increased foot stability after taping. Incidentally, many of the subjects in this study also reported feeling more "stable" after their foot was taped. Unfortunately, subject perception was not the focus of this study and therefore this information was not systematically solicited. The finding of this present study seems to imply that taping the foot in a bid to improve functional foot stability may be a placebo effect; the subjects felt more stable because they expected the foot taping to improve their foot stability although it, in actual fact, did not.

\subsection{The Effect of Low-Dye Taping on Running Gait}

The aim of low-Dye taping was to reduce rearfoot eversion, midfoot dorsiflexion and 1st toe dorsiflexion. The reverse- 6 tape strips around the calcaneus was meant to invert the heel and hold up the arch [18].

Looking at Table 2, the low-Dye taping significantly reduced rearfoot eversion during running. This showed that the reverse- 6 strips controlled the movement of the rearfoot effectively. The results of this present study agreed with that of Stoffel et al. [15] 
and Vicenzino et al. [16]. Analysing the data by groups, Fig. 5 showed that taping had the greatest effect in reducing the rearfoot eversion of the FFS group but no significant effect on the FFUS and NUS groups. This implies that to reduce rearfoot eversion by taping, feet that were functionally stable would benefit from taping whilst feet which were functional unstable would not. This is an unexpected finding as clinicians usually tape feet that were functionally unstable (FFUS and NUS groups). To validate this finding, future studies including a normal arched stable population should be included. This group was not included in this study as taping is rarely used in individuals with no structural or functional abnormalities.

The low-Dye taping reduced had no effect on midfoot dorsiflexion and 1st MPJ dorsiflexion (Table $2)$. Consistent results were also obtained when the results were analysed by groups (Figs. 6 and 7). The result of this study implied that low-Dye taping had no effect on the more distal parts of the foot. This finding was consistent with the findings of O'Sullivan et al. [17] who found that the main effect of low-Dye taping was to reduce rearfoot eversion movements only during walking.

This may indicate that the low-Dye taping technique may only be suitable for the management of certain running pathologies such as limiting tensile strain to the posterior tibial tendon caused by excessive rearfoot eversion. This taping technique will not help conditions such as plantar fasciitis, where the tensile stress is caused by excessive midfoot dorsiflexion and 1st MPJ dorsiflexion during running. Other modalities such as different taping techniques extending to the distal foot, or orthotic devices may need to be evaluated for its efficacy.

Analysing the data of the three groups, it is interesting to note that there is an interactive effect of taping on the loading rate between the three groups. Fig. 9 indicates that taping decreased the loading rate in the flat foot stable group but increased the loading rate in normal arched unstable group.

Many athletes with poor functional foot stability tape their feet so as to avoid foot related injuries during sports [9]. According to the results of this study, taping the flat foot that had good functional stability would be beneficial as loading rate was reduced. However, in individuals who have normal arched foot with poor functional stability, taping of the foot would increase the loading rate during running and potentially increase the risk of stress related running injuries. One limitation of this study was that only the ALD technique of taping was tested. Further studies comparing different taping techniques should be conducted to investigate if similar results would be obtained.

\section{Conclusion}

Taping significantly improved foot structure in individuals with flat feet. Taping did not improve functional foot stability. Taping reduced the amount of rearfoot eversion during running but had no effect on the more distal foot joints. The role of taping of the foot with rigid tape seems to be to provide an external rigid force to limit excessive movement. Taping the foot increased the loading rate of the normal arched functionally unstable foot. The practice of prophylactic foot taping in subjects with poor functional foot stability may reduce the risk of injuries due to excessive rearfoot movements, but may potentially increase the risk of impact injuries such as stress fractures. Thus, the use of taping should be cautioned.

\section{Acknowledgements}

We acknowledge the support of the following as contributions to the submission of this manuscript: Dr. Wong Khoon Yong who provided advice on the statistical analyses.

\section{References}

[1] Burns, J., Keenan, A., and Redmond, A. 2005. "Foot Type and Overuse Injury in Triathletes." Journal of the 
American Podiatric Medical Association 95 (3): 235-41.

[2] Ridder, R., Willems, T., and Roosen, P. 2012. "Plantar Pressure Distribution during Gait and Runnning in Subjects with Chronic Ankle Instability." Journal of Foot and Ankle Research 5 (Suppl 1): 32.

[3] Root, M., Orien, W., Weed, J., and Hughs, R., eds. 1971. Biomechanical Evaluation of the Foot. Los Angeles, CA: Clinical Biomechanics Corp.

[4] Freeman M., Dean, M., and Hanham, I. 1965. "The Etiology and Prevention of Functional Instability of the Foot." Journal of Bone and Joint Surgery 47 (4): 678-85.

[5] Hunt, A., and Smith, R. 2004. "Mechanics and Control of the Flat versus Normal Foot during Stance Phase of Walking." Clinical Biomechanics 19 (4): 391-7.

[6] Horak, F., and Nasher, L. 1986. "Central Programming of Postural Movements: Adaptation to Altered Support Surface Configurations." J. Neurophysiol. 55 (6): 167-77.

[7] Pfeiffer, R., and Mangus, B., eds. 2012. Concepts of Athletic Training. London, UK: Jones and Bartlet Publishers International, 254.

[8] Herradine, P., Herrington, L., and Wright, R. 2001. "The Effect of Low-Dye Taping upon Rearfoot Motion and Position before and after Exercise." The Foot 11 (2): 57-60.

[9] Cornwall, M., McPoil, T., and Fair, A. 2014. "The Effect of Exercise and Time on the Height and Width of the Medial Longitudinal Arch Following the Modified Reverse-6 and the Modified Augmented Low-Dye Taping Procedures." The International Journal of Sports Physical Therapy 9 (5): 635-43.

[10] Luque-Suarez, A., Gijon-Nogueron, G., Baron-Lopez, F., Labajos-Manzanares, M., Hush, J., and Hancock, M. 2013. "Effects of Kinesiotaping on Foot Posture in Participants with Pronated Foot: A Quasi-randomised, Double-Blind Study." Physiotherapy 100 (1): 36-40.

[11] Miralles, L., Monterde, S., Montull, S., Salvat, I., Fernandez-Ballart, J., and Baceiro, J. 2010. "Ankle Taping Can Improve Procrioception in Healthy Volunteers." Foot Ankle Int. 31 (12): 1099-106.

[12] Robbins, S., Waked, E., and Rappel, R. 1995. “Ankle Taping Improves Proprioception before and after Exercise in Young Men.” Br. J. Sports Med. 29 (4): 242-7.

[13] Delahunt, E., McGrath, A., Doran N., and Coughlan, G. 2010. "Effect of Taping on Actual and Perceived Dynamic Postural Stability in Persons with Chronic Ankle Instability." Arch. Phys. Med. Rehabilitation 91 (9): 1383-9.

[14] Papadopoulos, E., Nikolopoulos, C., Badekas, A., Vagenas, G., Papadakis, S., and Athanasopoulos, S. 2007. "The Effect of Different Skin-Ankle Brace Application Pressure in Quiet Single Limb Balance and
Electromyographic Activation Onset of Lower Limb Muscles." BMC Musculoskeletal Disorders 8 (Sep.): 89.

[15] Stoffel, K., Nicholls, R., Winata, A., Dempsey, A., Boyle, J., and Lloyd, D. 2010. "Effect of Ankle Taping on Knee and Ankle Joint Kinematics in Sporting Tasks.” Med. Sci. Sports Exerc. 42 (11): 2089-97.

[16] Vicenzino, B, McPoil, T., and Buckland, S. 2007. "Plantar Foot Pressures after the Augmented Low-Dye Taping Technique." J. Athl. Train. 42 (3):374-80.

[17] O' Sullivan, K., Kennedy, N., O’Neill, E., and Ni Mhainin, U. 2008. "The Effect of Low-Dye Taping on Rearfoot Motion and Plantar Pressure during Stance Phase of Gait." BMC Musculoskeletal Disorders. Assessed January $15,2014$. http://www.biomedcentral.com/1471-2474/9/111.

[18] Carter, K., and Chockalingam, N. 2009. “An Assessment of Strapping Techniques Commonly Used for Pronated Foot Deformities." J. Am. Podiatr. Med. Assoc. 99 (5): 391-8.

[19] Keenan, A., Redmond, A., Horton, M., Conaghan, P., and Tennant, A. 2007. "The Foot Posture Index: Rasch Analysis of a Novel Foot Specific Outcome Measure." Arch. Phys. Med. Rehabil. 88 (1): 88-93.

[20] Redmond, A., Crosbie, J., and Ouvrier, R. 2006. "Development and Validation of a Novel Rating System for Scoring Standing Foot Posture, the Foot Posture Index." Clinical Biomechanics 21 (1): 89-98.

[21] Sánchez-Rodríguez, R., Martinez-Nova, A., Escamillia-Martinez, E., and Pedrera-Zamorano J. D. 2012. "Can the Foot Posture Index or Their Individual Criteria Predict Dynamic Plantar Pressures?" Gait and Posture 36 (3): 591-5.

[22] Redmond, A. 2005. The Foot Posture Index User Guide and Manual. Accessed November 13, 2013.http://www.leeds.ac.uk/medicine/FASTER/z/pdf/FP I-manual-formatted-August-2005v2.pdf.

[23] Bell, D., Guskiewicz, K., Clark, M., and Padua, D. 2011 "Systemic Review of the Balance Error Scoring System." Sports Health 3 (3): 287-95.

[24] Ross, S., Linens, S., Wright, C., and Arnold, B. 2011. "Balance Assessments for Predicting Functional Ankle Instability and Stable Ankles." Gait and Posture 34 (4): 539-42.

[25] Arnold, B., de la Motte, S., Linens, S., and Ross, S. 2009. "Ankle Instability Is Associated with Balance Impairments: A Meta-analysis." Med. Sci. Sports Exerc. 41 (5): 1048-62.

[26] Kirtley, C. 2006. Measurement of Gait Kinematics, Clinical Gait Analysis: Theory and Practice. London, United Kingdom: Churchill Livingtone, 44-5.

[27] Wilken, J., Rao, S., Saltzman, C., and Yack, J. 2011. "The Effect of Arch Height on Kinematic Coupling 
during Walking." Clinical Biomechanics 26 (3): 318-23.

[28] Logan, S., Hunter, I., Hopkins, J., Feland, J., and Parcell, A. 2010. "Ground Reaction Force Differences between Running Shoes, Racing Flats, and Distance Spikes in Runners." Journal of Sports Science and Medicine 9 (1): 147-53.

[29] Zadpoor, A. A., and Nikooyan, A. A. 2011. "The Relationship between Lower Extremity Stress Fractures and Ground Reaction Force: A Systematic Review."
Clinical Biomechanics 26 (1): 23-8.

[30] Wilfried, A., Lohrer, H., and Gollhofer, A. 1999. "Functional Properties of Adhesive Ankle Taping: Neuromuscular and Mechanical Effects before and after Exercise." Foot Ankle Int. 20(4): 238-45.

[31] Halseth, T., McChesney, J., DeBeliso, M., Vaughn, R., and Lien, J. 2004. "The Effects of Kinesio"T Taping on Proprioception at the Ankle." J. Sports Sci. Med. 3 (1): $1-7$. 